\title{
Chaos controller for switching regulators aiming enhanced design-space towards miniaturization
}

\author{
E. Rodriguez ${ }^{\dagger}$, A. El Aroudi ${ }^{\ddagger}$, H. Martinez ${ }^{\dagger}$, A. Poveda ${ }^{\dagger}, \mathrm{H} \mathrm{Iu}^{\diamond}$ and E. Alarcon ${ }^{\dagger}$ \\ $\uparrow$ School of Electrical, Electronic and Computer Engineering, \\ UPC BarcelonaTech \\ 08034 Barcelona, Spain. \\ $\ddagger$ Departament d'Enginyeria Electrnica, Elctrica i Automtica, \\ Universitat Rovira i Virgili \\ 43007 Tarragona, Spain \\ ${ }^{\diamond}$ School of Electrical, Electronic and Computer Engineering, \\ University of Western Australia
}

\begin{abstract}
This paper tackles the control of fast-scale instabilities in a buck switching power converter aiming to expand its design-space towards miniaturization. After briefly revisiting the working principle of existing chaos controllers, the paper explores an alternative approach based on amplifying the harmonic at the switching frequency. Numerical simulations show that the proposed controller can concurrently improve both fast-scale and slow-scale stability margins. Finally, the paper proposes a chaos controller combined with an output ripple reduction network and studies their interaction with the aim of achieving both low-ripple and improved stability.
\end{abstract}

\section{Introduction}

Switching power converters are widely used in many applications due to their high efficiency, small size and low weight. However, the design-space when targeting miniaturization towards on-chip integration is technologically limited by three performance metrics: area, efficiency and output voltage ripple. The area occupancy is mainly limited by the reactive components size; thus, reducing the inductor or capacitor parameter values results in a decrease of the area, but also increases the natural frequency of the implicit low-pass filter of any DC-DC converter, turning into the reduction of the ratio between the switching frequency and the natural frequency hence leading to high output voltage ripple. This ripple is usually decreased by increasing the switching frequency, hence, in turn, decreasing the overall converter efficiency. Therefore, the desired low-area highefficiency context, such as in miniaturized switching power converters, will upfront imply high output voltage ripple.

However, the output voltage ripple amplitude confronts a two-fold limitation: due to the strict specifications imposed by on-chip loads demanding tight regulation, but also because higher output voltage ripple make the converter more prone to exhibit the so-called fast-scale instabilities (FSI) and eventually chaotic regime [1].

The control of FSI, manifested by subharmonic oscillation, has been traditionally tackled either by adding external signals such as ramp compensation in current-mode control strategies in power electronics circuits, or adding a time-delay module in general physical nonlinear dynamical systems[2]. In this second case, in addition to their implementability constraints, in [3] it is shown that these controllers are based on comb filters centered at half of the switching frequency and that the global stability boundary is compromised due to their additional phase lag in the system loop hence worsening the well-known slow-scale instability (SSI) boundary.

On the other hand, the reduction of the converter output ripple has been usually tackled by relaxing circuit specifications (increase reactive components values or switching frequency), or by using more complex topologies such as multilevel converters [4], or by narrowband reactive filtering at the converter output [5].

The system considered in this paper is shown in Fig. 1 and the parameter values used are $V_{g}=6 \mathrm{~V}, V_{\text {ref }}=3 \mathrm{~V}$, $R=2.5 \Omega, L=66 \mathrm{nH}, C=20 \mathrm{nF}, f_{s}=50 \mathrm{MHz}, V_{m}=$ $1 \mathrm{~V}, \omega_{z 1}=10 \mathrm{Mrad} / \mathrm{s}$ and $k_{p}=3$. These values correspond to a miniaturized converter aiming on-chip integration [4], but the selection is representative (through scaling) of any converter exhibiting moderately large ripple $(\sim 50 \mathrm{mV})$.

The remaining of the paper is structured as follows. First, Section II reviews briefly the time delay controller and explores the so-called repetitive controller showing that not only the attenuation at half of the switching frequency but also amplifying the harmonic at the switching frequency can entail the control of FSI. Then, a new controller is proposed, which is based on a narrow band amplifier (NBA) centered at the switching frequency improving the stability boundary in both FSI and SSI terms, apart from simplifying the implementability of the controller. The paper concludes by proposing a new topology, so-called LC 


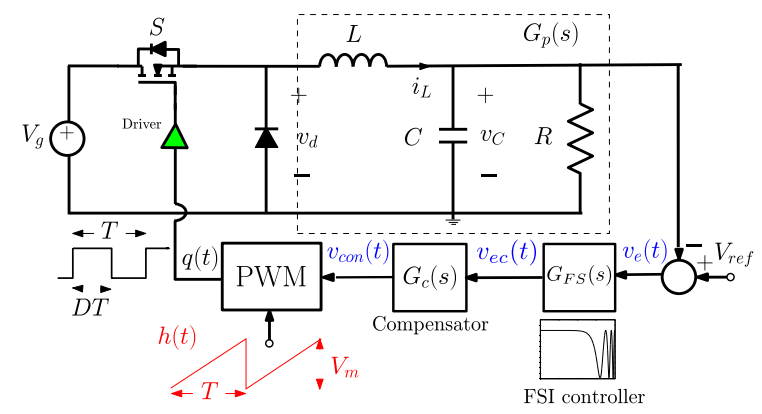

Figure 1: VMC buck converter with a compensator $G_{c}(s)$ along with a FSI controller $G_{F S}(\mathrm{~s})$.

divider, in which the feedback ripple component is amplified to obtain an enhanced stability margin while attenuating the converter output voltage in order to accomplish the load ripple specifications.

\section{Generalized time delay feedback chaos controllers: Repetitive controllers}

Reference [3] revisits time-delay feedback controllers (TDFC) in order to describe their operation principle and the effect of their parameters upon stability. The work shows that the controller provides a frequency-selective comb-filter which attenuates the harmonics at half of the switching frequency and its integer multiples.

However, other more complex time-delay-based controllers have been also used for harmonic tracking applications such as the so-called repetitive controller [6]. The block diagram of a repetitive controller is shown in Fig. 2. The $s$-domain transfer function of such a repetitive controller in terms of the parameter $\gamma$ is:

$$
G_{F S}(s)=\frac{e^{-s T}+\gamma}{e^{-s T}-\gamma}
$$

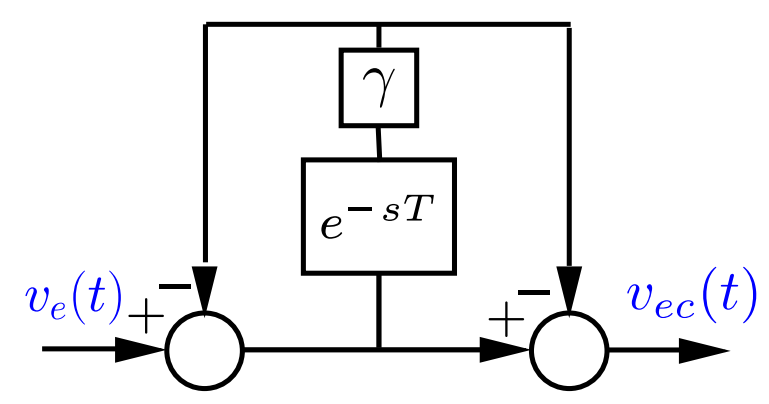

Figure 2: Block diagram of a repetitive controller.

The frequency response of a repetitive controller is shown in Fig. 3 along with that of TDFC. Note that comparing with a simple TDFC, apart from attenuating the harmonic at half of the switching frequency, the controller is
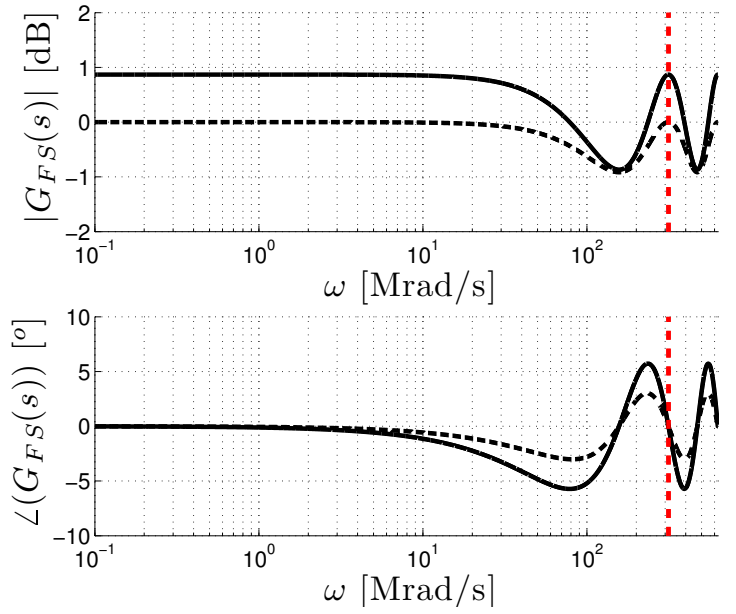

Figure 3: Bode diagram representation (magnitude and phase) of the repetitive controller with $\gamma=0.05$ (solid) and TDFC (dash). Vertical dashed line indicates the switching frequency.

amplifying the switching frequency harmonics (to improve the tracking performance).

The stability boundary as a function of the proportional gain $k_{p}$ is depicted in Fig. 4, showing that the system under the repetitive controller is less stable in terms of SSI but it is slightly more stable in terms of FSI. Regarding the SSI observed results, they agree with the frequency domain interpretation, in which more phase lag is added by the controller. However, in terms of FSI, the improvement of the stability margin compared to TDFC can be only attributed to the amplification at the switching frequency harmonic.

\section{Narrow band amplifier controller}

The previous section suggests that FSI control can be accomplished not only by attenuating the harmonic at half of the switching frequency but also amplifying the switching frequency harmonic. Thus, this section focuses on providing the FSI control functionality from a feasible implementation of such harmonic amplification.

The transfer function of the FSI chaos controller $G_{F S \text {,NBA }}(s)$ based on a NBA is shown in Eq. 2 with $\Delta \xi=$ $\xi_{1} / \xi_{2}>1$ and the center frequency tuned to the switching frequency, $\omega_{n}=2 \pi f_{s}$. Apart from the amplification at the switching frequency harmonic, a key additional advantage is the fact that it notably improves the SSI boundary by adding a phase lead before the switching frequency.

$$
G_{F S, \mathrm{NBA}}(s)=\frac{s^{2}+2 \xi_{1} \omega_{n} s+\omega_{n}^{2}}{s^{2}+2 \xi_{2} \omega_{n} s+\omega_{n}^{2}}
$$

Its effect upon FSI boundary is characterized in Fig. 6, which shows the stability boundaries surface as a function of $\Delta \xi, \xi_{2}$ and converter parameters. These surfaces 

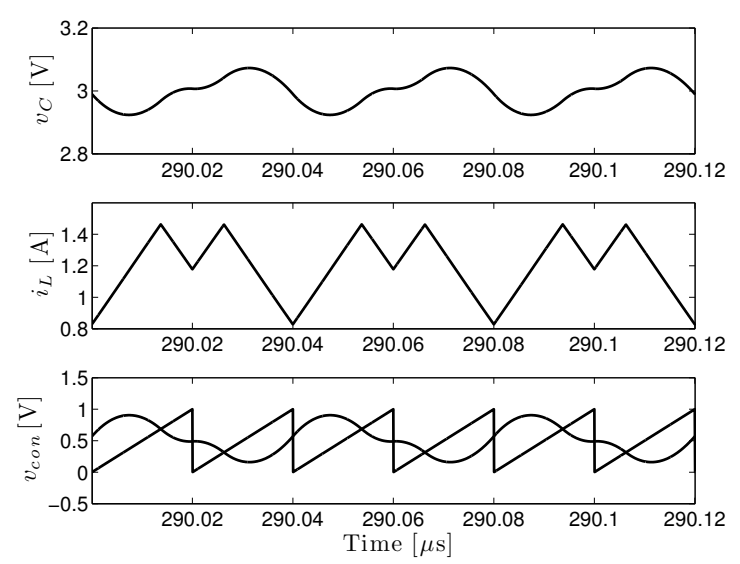

(a)
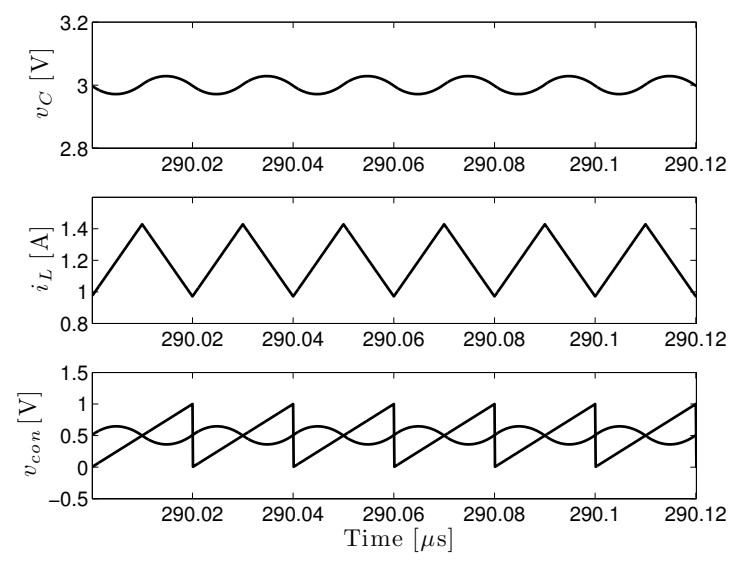

(b)

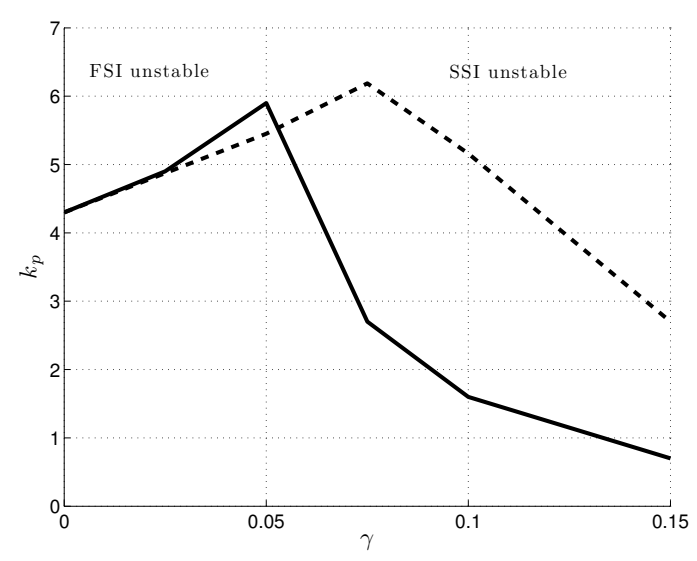

(c)

Figure 4: Time domain wavforms with $k_{p}=5$ (a) without FSI controller (b) with Repetitive controller with $\gamma=0.05$. (c) Stability boundary as a function of $\gamma$ and $k_{p}$ with a repetitive controller (solid) and TDFC (dashed line). FSI stability boundary without controller $(\gamma=0), k_{p}=4.3$.

have been obtained from the discrete-time model $[7,8]$ of the system, the well-known complex mathematical model
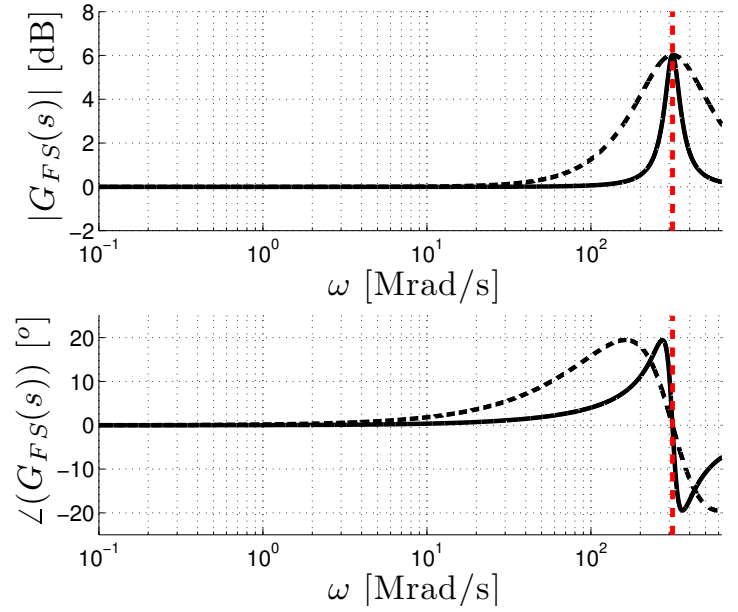

Figure 5: Bode diagram representation (magnitude and phase) of an NBA tuned to the switching frequency (vertical dashed-line) with $\Delta \xi=2$ and $\xi_{2}=0.1$ (solid) and $\xi_{2}=0.5$ (dash).

commonly used to predict subharmonic oscillation boundary with high accuracy. Note that depending upon the value of the two parameters, the obtained results are much more better than the one obtained in delay-based controllers (see Fig. 4).

As it was mentioned at the beginning of this work, the indirect aim of a chaos controller, apart from obviously improving the stability margin, is that such improvement allows to expand the design-space towards miniaturization (for instance reducing the inductance value, which is related to the system area), without losing stability.

Simulations in Fig. 6 show the stability boundary as a function of $\Delta \xi$ and $\xi_{2}$ and the inductance (related to area/volume occupancy) and switching frequency (related to efficiency).

Both obtained surfaces are similar and the stability boundary is clearly improved, allowing to reduce both parameter values without losing stability, when $\xi_{2}<1$ and $\Delta \xi>1$ (a narrow band amplification) with regards to the case of not using such controller $(\Delta \xi=1)$. Note that the parameter $\xi_{2}$, which has a direct effect upon the width of the amplification band, has an important effect upon the stability boundary so that as it increases, the FSI boundary is worsened. This suggests that the benefits of the NBA controller on stability are limited by such bandwidth, namely when both harmonics at the switching frequency and its half are amplified the FSI boundary is not modified. On the other hand, it is possible to observe that the higher the amplification is, the more stable the system is in terms of FSI. 


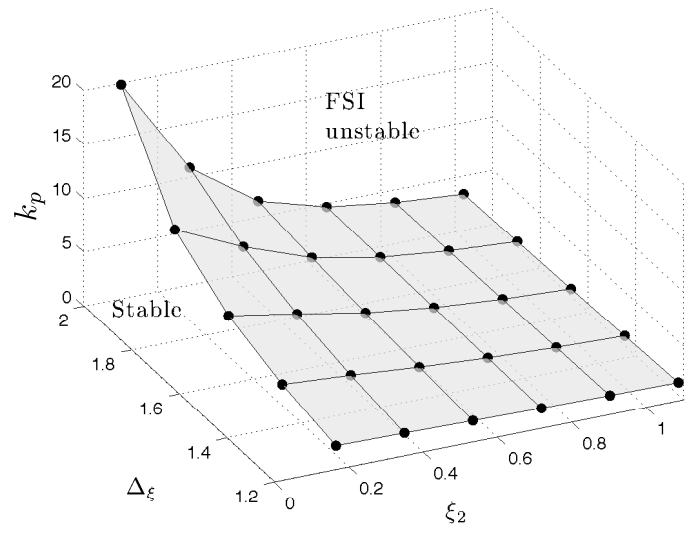

(a)

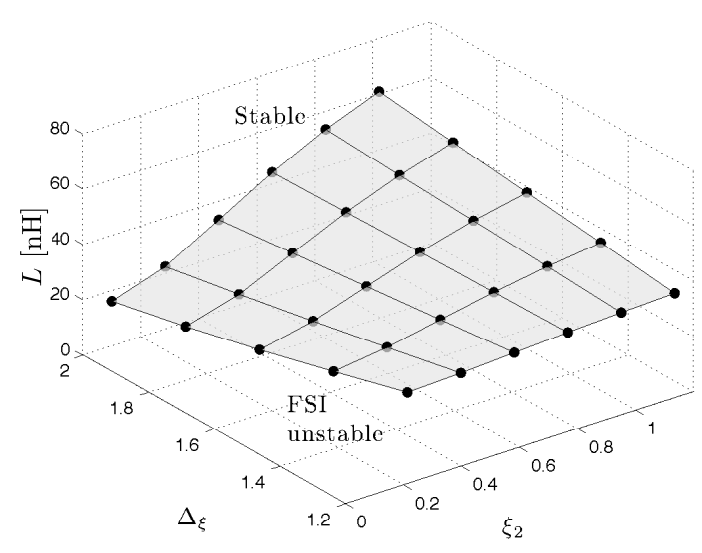

(b)

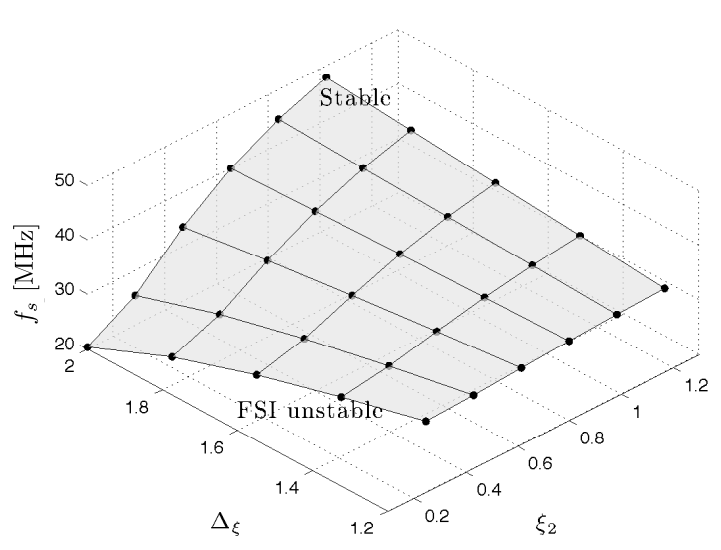

(c)

Figure 6: FSI boundary surfaces, obtained from the discrete-time model, of a VMC buck converter with an NBA as a function of parameters $\Delta \xi, \xi_{2}$ and (a) the proportional gain $k_{p}$, (b) the inductance $L$ and (b) the switching frequency $f_{s}$.

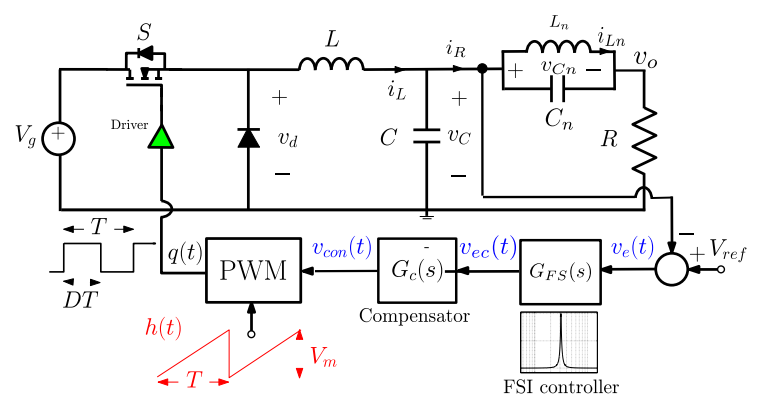

(a)

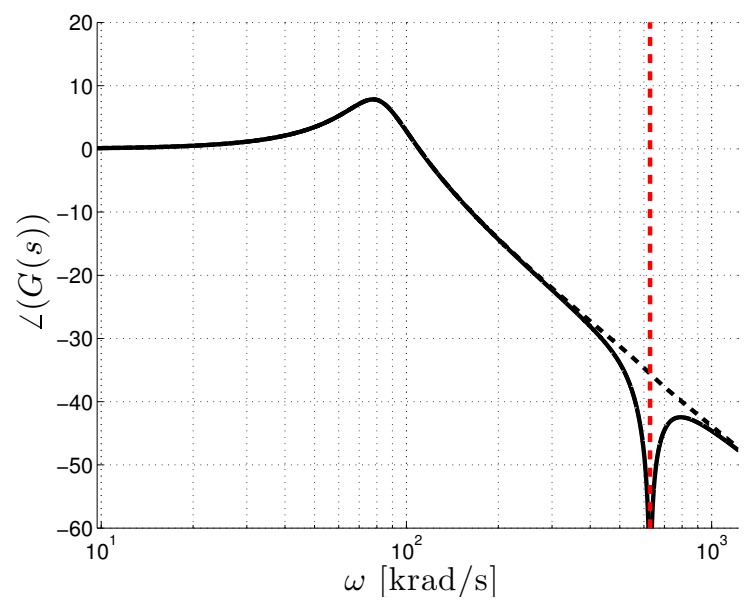

(b)

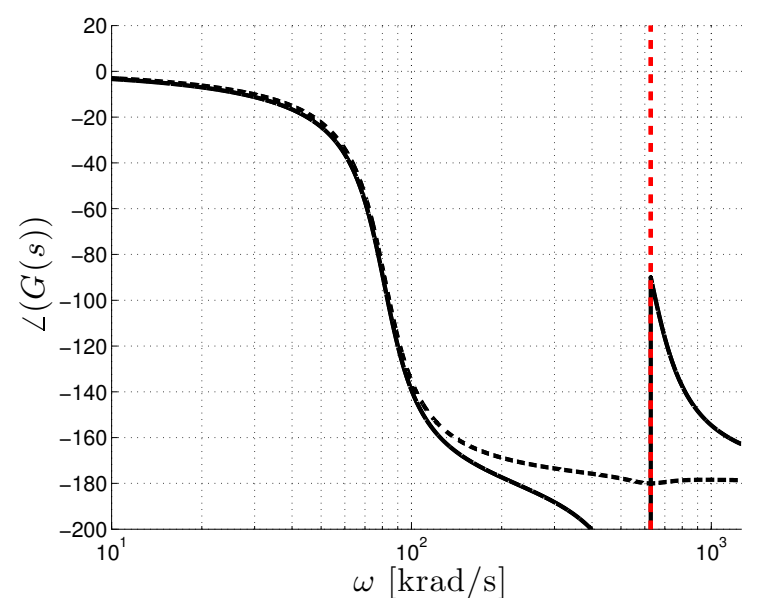

(c)

Figure 7: (a) Frequency response of the buck switching converter under voltage-mode control with a PWM and an additional NBA controller and output notch filter. (b) Magnitude and (c) phase of the control-to-output (solid) and control-to-feedback (dashed) transfers function with the output notch filter. Vertical dashed-line indicates the switching frequency. 


\section{Combining chaos controller with output ripple re- duction}

The improvement in stability terms when a chaos controller is used implies a clear step forward towards circuit miniaturization without losing stability. However, this will imply an increase in the converter output ripple, which is not desirable in order to keep the DC-DC nature of the system. Therefore, the advantage of the NBA chaos controller could be invalidated because of the expansion of the design-space, namely the area or switching frequency reduction could be limited by load specifications in terms of ripple.

The natural way for tackling this excess of ripple would be to add a ripple attenuation module to the converter output, but this is not trivial since this should be based on modifying the switching frequency harmonic, which is the same working principle of the NBA chaos controller, and hence it could cancel the advantage of such approach.

This section proposes a joint approach to obtain high stability margin in term of FSI, that allows reducing the converter reactive components, and at the same time reducing the output voltage ripple to accomplish low-ripple performance as required by DC-DC converters.

The frequency domain representation of the buck switching converter under voltage-mode control with a PWM and an additional NBA controller and output notch filter are depicted in Fig. 7. Both the control-to-feedback and the control-to-output transfer functions show that the output of the LC network does not affect the feedback transfer function but adds a notch in the output transfer function hence reducing the amount of ripple delivered to the load. Additionally the NBA will be added as a chaos controller in the feedback loop.

In Fig. 8 results from using only the NBA (obtained in the last section) and this approach are compared. The results are obtained from the discrete-time model developed. Note that a similar stability boundary is obtained as a function of the duty cycle, but the output voltage ripple is largely reduced by canceling the harmonic at the switching frequency.

The main drawback of this proposed configuration is its additional required circuitry: the NBA plus the reactive components of the output notch. In addition, the notch inductor should drive all the output current hence requiring large area and the circuit also requires accurately controlling the center frequency and the gain of the amplifier and the output notch since a mismatch between both center frequencies (such as those due to the effect of temperature or parasitic elements) could limit the benefits of the approach in both output voltage ripple and fast-scale stability terms.

\section{Conclusions}

This work has addressed controllers for fast-scale instability (FSI) in a buck switching power converter in pur-

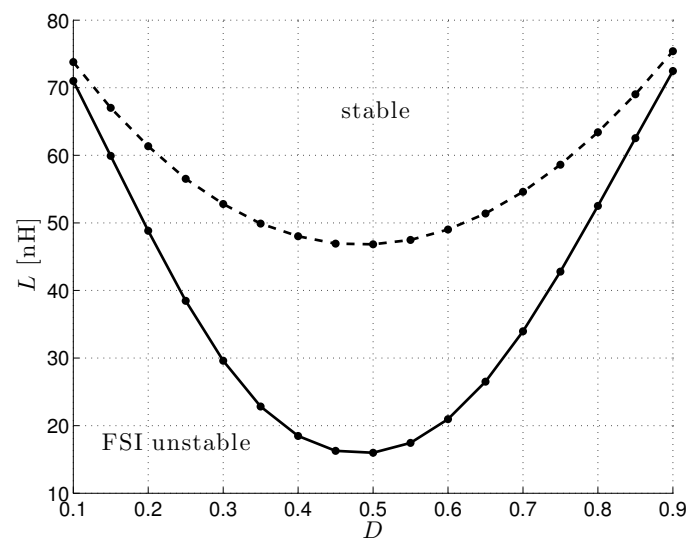

(a)

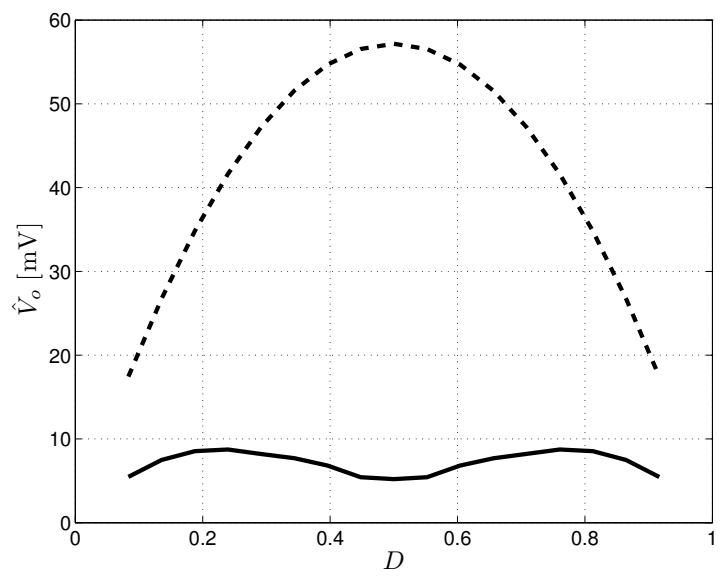

(b)

Figure 8: (a) FSI boundary curves, obtained from the discrete-time model, of a VMC buck converter with a NBA controller and output notch filter (solid) and a conventional buck (dash) as a function of the inductance $L$ and the duty cycle $D$ and (b) the output voltage ripple $\hat{V}_{o}$ in both cases as a function of the duty cycle $D . L=66 \mathrm{nH}, L_{n}=2 \mathrm{nH}$ $C_{n}=5 \mathrm{nF}, \xi_{2}=0.1$ and $\Delta \xi=2$.

suit of expanding the design-space when targeting miniaturization. The control of such instabilities would allow the reduction of reactive component values or the switching frequency without losing stability. With this purpose, this paper has proposed a FSI controller based on a narrow band amplifier (NBA) centered at the switching frequency harmonic which does not compromise the global stability boundary. A final extension has been considered to combine the NBA controller with an output ripple reduction network in the power topology with the aim of concurrently achieving a low-ripple at the output of the converter and an improved global stability of the closed loop system. 


\section{Acknowledgments}

This work is funded by FPU-grant of ministry of Science and Innovation of Spain under grants DPI2010-16481 and TEC2010-15765.

\section{References}

[1] Rodriguez, A. E. Aroudi, F. Guinjoan, and E. Alarcon, "A ripple-based design-oriented approach for predicting fast-scale instability in DC-DC switching power supplies," IEEE Transactions on Circuits and Systens I: Regular Papers, vol. 59, pp. 215-227, 2012.

[2] K. Pyragas, "Continuous control of chaos by selfcontrolling feedback," Physics Letters A, vol. 170, pp. 421-428, 1992.

[3] E. Rodriguez, E. Alarcn, H. Iu, and A. El Aroudi, "A frequency domain approach for controlling chaos in switching converters," in IEEE International Symposium on Circuits and Systems (ISCAS'10), 2010, pp. 2928-2931.

[4] G. Villar and E. Alarcon, "Monolithic integration of a 3-level DCM-operated low-floating-capacitor buck converter for DC-DC step-down donversion in standard CMOS," in IEEE Power Electronics Specialists Conference (PESC'08), 2008, pp. 4229-4235.

[5] E. Alarcon, G. Villar, S. Ferrandez, F. Guinjoan, and A. Poveda, "Ripple-reduction tuned filtering switching power converter topology," in IEEE Power Electronics Specialists Conference (PESC'04), 2004.

[6] G. Escobar, P. Martinez, J. Leyva-Ramos, and P. Mattavelli, "A negative feedback repetitive control scheme for harmonic compensation," IEEE Transactions on Industrial Electronics, vol. 53, no. 4, pp. 1383 -1386, 2006.

[7] M. di Bernardo and F. Vasca, "Discrete-time maps for the analysis of bifurcations and chaos in DC/DC converters," IEEE Transactions on Circuits and Systems I: Fundamental Theory and Applications, vol. 47, no. 2, pp. 130-143, 2000.

[8] A. El Aroudi, M. Debbat, L. Giral, G. Olivar, L. Benadero, and E. Toribio, "Bifurcations in DC-DC swithching converters: reivew of methods and applications," International Journal of Bifurcation and Chaos, vol. 15, no. 5, pp. 1549-1578, 2005. 